\title{
EXISTENCE OF SOLUTIONS FOR SOME NONCOERCIVE ELLIPTIC PROBLEMS INVOLVING DERIVATIVES OF NONLINEAR TERMS
}

\author{
Lucio BocCARdo, Gisella CROCE AND Luigi OrsinA
}

Abstract. We study a nonlinear equation with an elliptic operator having degenerate coercivity. We prove the existence of a $W_{0}^{1,1}(\Omega)$ solution which is distributional or entropic, according to the growth assumptions on a lower order term in divergence form.

Mathematics subject classification (2010): 35B45, 35D05, 35D10, 35J65, 35J70.

Keywords and phrases: degenerate ellipticity, boundary value problem, distributional solutions, entropy solutions.

\section{REFERENCES}

[1] Ph. Bénilan, L. Boccardo, T. Gallouët, R. Gariepy, M. Pierre and J.L. Vázquez, An $L^{1}$-theory of existence and uniqueness of solutions of nonlinear elliptic equations, Ann. Scuola Norm. Sup. Pisa Cl. Sci., 4 (1995), 241-273.

[2] L. Boccardo, Some nonlinear Dirichlet problems in $L^{1}$ involving lower order terms in divergence form, Progress in elliptic and parabolic partial differential equations (Capri, 1994), 43-57, Pitman Res. Notes Math. Ser., 350, Longman, Harlow, 1996.

[3] L. Boccardo, H. Brezis, it Some remarks on a class of elliptic equations, Boll. Unione Mat. Ital., 6 (2003), 521-530.

[4] L. Boccardo, G. Croce, L. ORsina, Nonlinear degenerate elliptic problems with $W_{0}^{1,1}$ solutions, to appear in Manuscripta Math.

[5] L. Boccardo, D. Giachetti, J. I. Díaz, F. Murat, Existence and regularity of renormalized solutions for some elliptic problems involving derivatives of nonlinear terms, J. Differential Equations, 106 (1993), 215-237.

[6] L. Boccardo, A. Dall' Aglio, L. ORsina, Existence and regularity results for some elliptic equations with degenerate coercivity, dedicated to Prof. C. Vinti (Perugia, 1996), Atti Sem. Mat. Fis. Univ. Modena, 46 (1998), 51-81.

[7] G. CROCE, The regularizing effects of some lower order terms in an elliptic equation with degenerate coercivity, Rendiconti di Matematica, 27 (2007), 299-314.

[8] G. Dal Maso, F. Murat, L. Orsina, A. Prignet, Renormalized solutions for elliptic equations with general measure data, Ann. Scuola Norm. Sup. Pisa Cl. Sci., 28 (1999), 741-808.

[9] J. LENNON, P. MCCARTNEY, In my life, Rubber Soul, 1965. 